\section{Varieties of Religious Coping: Exploratory and Factor Analysis of Religious Coping Scale in Midlife in the United States Survey}

Joe Chelladurai

\title{
Participants
}

For this study, I used data from the 2005 Midlife in the United States (MIDUS) survey. Participants in the second wave (2005) included 1490 individuals.

\section{Measures}

Religious coping was measured based on the following set of eight items:

1. How often do you seek comfort through religious or spiritual means... ?

2. When you have decisions to make in your life, how often do you ask yourself what your religious or spiritual beliefs suggest you should do?

3. I try to make sense of the situation and decide what to do without relying on God.

4. I wonder whether God has abandoned me.

5. I feel God is punishing me for my sins or lack of spirituality

6. I look to God for strength, support, and guidance.

7. I work together with God as partners.

8. I think about how my life is part of a larger spiritual force.

Although in recent decades religion is reportedly in a declining trend, people still turn to religion in times of stress and challenges (Hood, Hill, \& Spilka, 2018). People cope differently with religion as theorized by Pargament and colleagues (1998) and may have various coping styles categorized broadly as positive and negative coping.

Positive religious coping takes the form of participation in religion through seeking support and redefining stressors in a positive light. For instance, people may view their stressors as beneficial, although challenging at present, and that God will help them get through. On the other hand, people may engage in negative coping behaviors that reframe challenges as detrimental. For example, when people consider themselves as deserving punishment from God, they may cope negatively.

In this study, using exploratory factor analysis, I examined the factor structure of the religious coping measure in the Midlife in the United States Survey. Drawing from previous research, I hypothesized that religious coping would have at least two underlying factors. Exploratory factor analysis was also used as a dimension reduction technique to refine and shorten the number of questions required to capture the desired phenomenon. EFA was used to assess whether the construct was uni-dimensional or multi-dimensional. The underlying factor structures were examined using factor analysis procedures including factor rotation, eigenvalue thresholds, and scree plots.

\section{Analysis Plan}

I examined the religious coping measure and tested whether the measure loaded on a single factor unidimensional scale or two-factor multidimensional scale. I used psych and GPArotation packages in $\mathrm{R}$ to perform the analyses.

\section{Results}

A factor analysis was conducted with the eight items of religious coping. The third item, "I try to make sense of the situation and decide what to do without relying on God" was omitted from further analysis as the factor loading was .40.

Results of the one factor solution omitted items 4 and 5 and presented loadings for the remaining items. This suggests that the omitted items may load on to a different factor.

Table 1. One Factor Solution of Religious Coping

\begin{tabular}{cr}
\hline & Factor 1 \\
\hline Item 1 & 0.829 \\
Item 2 & 0.837 \\
Item 4 & \\
Item 5 & \\
Item 6 & 0.903 \\
Item 7 & 0.873 \\
Item 8 & 0.709 \\
\hline
\end{tabular}




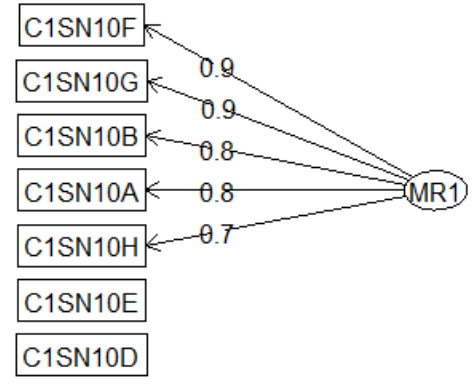

Figure 1. Factor Analysis Diagram (unidimensional)

As indicated by the above diagram, items 4 and 5 as represented by original variable codes (C1SN10E and C1SN10D) were omitted in the model.

\section{Eigenvalues}

By examining eigenvalues using a scree plot it was indicative of a two-factor structure of religious coping. The eigen values were $3.80,1.54,0.50,0.44,0.35,0.21$, and 0.13 . As two values were above the cutoff value of 1 , there was a strong indication of at least two factors in the religious coping scale.

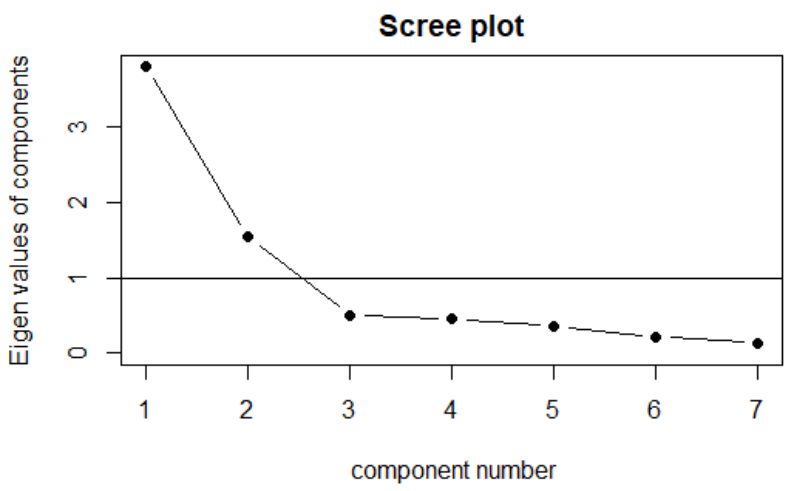

Next, a factor analysis model specified with two factors was identified. Results confirmed the previous indication of two factors. Items 4 and 5 loaded on factor 2 .

Table 2. Two-Factor Solution

\begin{tabular}{lrr}
\hline & Factor 1 & Factor 2 \\
\hline Item 1 & 0.839 & \\
Item 2 & 0.851 & \\
Item 4 & & 0.547 \\
Item 5 & & 0.997 \\
Item 6 & 0.905 & \\
Item 7 & 0.885 & \\
Item 8 & 0.7 & \\
\hline
\end{tabular}

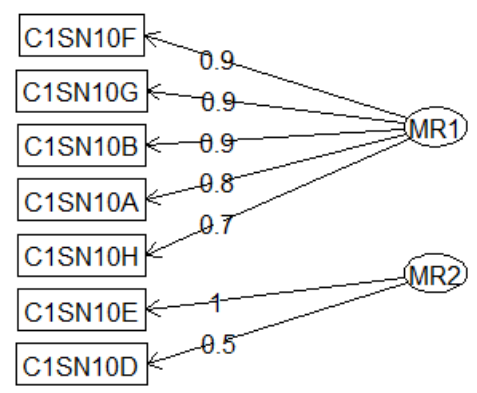

Figure 2. Factor Analysis Diagram (multidimensional)

As illustrated by the above diagram, two factors were identified suggesting multi-dimensionality of the religious coping measure.

\section{Discussion}

Results from the analysis indicated that the religious coping measure has a two-factor structure. Based on the items, the exploratory factor analysis provided empirical verification of Pargament's religious coping theory. In this brief version of the scale, there were two factors representing positive and negative religious coping. Exploratory factor analysis provided a reliable connection between theory and data based on respondent's experiences.

This finding supports previous research examining the positive and negative styles of religiosity. There are however limits to a dichotomous classification of religious coping. It may be true that participants may rate themselves higher on both positive and negative coping styles. Future research may examine such complexities of how people cope and navigate through life's challenges and how religion helps or hinders their journey.

\section{References}

Hood Jr, R. W., Hill, P. C., \& Spilka, B. (2018). The psychology of religion: An empirical approach. Guilford Publications.

Pargament, K. I., Smith, B. W., Koenig, H. G., \& Perez, L. (1998). Patterns of positive and negative religious coping with major life stressors. Journal for the Scientific Study of Religion, 710-724. 